\title{
Adaptable and Automated Rodent Behavior Maze System
}

Jacob M Olson ${ }^{1, *}$, Christopher A Leppla1, *, John H Bladon¹, Shantanu P Jadhav ${ }^{1}$

1 - Neuroscience Program, Department of Psychology and Volen National Center for Complex Systems, Brandeis University, Waltham, MA, 02453, USA

* - These authors contributed equally.

\section{Highlights}

- Modular maze system enables same-day behavior recordings from multiple flexibly-configured maze environments.

- High degree of experimental reproducibility and repeatability.

- Automated lick-detection, liquid reward delivery, and movable barriers.

- Electronics interface with SpikeGadgets hardware for plug-and-play automated control.

- Flexibility enables rapid experimental piloting.

\begin{abstract}
Mazes are a fundamental and widespread tool in behavior and systems neuroscience research in rodents. However, their form and inflexibility often restrict potential experimental paradigms that involve multiple or adaptive maze designs. Unique layouts often cost substantial engineering and time investments from trainee scientists. To alleviate these issues, we have developed an automated modular maze system that is flexible and scalable. This system will allow for experiments with multiple track configurations in rapid succession. Additionally, the flexibility can expedite prototyping of behaviors. Finally, the standardized componentry enhances experimental reproducibility and repeatability. This maze system presents advantages over current maze options and can facilitate novel behavior and systems neuroscience research.
\end{abstract}




\section{Introduction}

Rodent navigation of mazes is a longstanding and widespread method for investigating behavior, cognitive processes, and neurophysiology. This experimental technique leverages the fact that navigating to rewards or away from danger is a natural task for both rats and mice. Beginning over a century ago at the advent of the $20^{\text {th }}$ century (Small, 1901), maze experiments have resulted in landmark discoveries regarding cognitive processes of learning \& memory underlying navigation (Tolman et al., 1946) and its neural substrates (O'Keefe \& Dostrovsky, 1971; Olton \& Samuelson, 1976). Currently, standard maze designs are pervasive and underlie foundational tasks in learning and memory, decision-making, and even anxiety (e.g. Barnes, 1979; Frank et al., 2000; Handley \& Mithani, 1984; Morris et al., 1982; O’Keefe \& Dostrovsky, 1971; Olton \& Samuelson, 1976). In addition, unique mazes are constantly being designed to test specific questions in these fields (Ainge et al., 2007; Böhm \& Lee, 2020; Knierim et al., 2000; Nitz, 2006; Olson et al., 2017; Porter et al., 2018; Steiner \& Redish, 2014; Tanila et al., 2018; Wilson et al., 2015). Looking forward, the ability to rapidly switch environments while monitoring neural activity continuously is a growing desire in systems neuroscience to investigate contextual memory and representations, spatial remapping, flexible and adaptive decision making. The explosion of neural data recording capabilities and advances in machine learning techniques for analysis further support the value of increasing complex behavioral datasets.

Current maze designs limit the ability for multiple maze environment recordings as part of one experiment. For simple, less automated mazes, each maze is often one piece, with limited or no flexibility for other maze configurations. If multiple maze configurations are desired, they must all be made and stored, and then moved into and out of place in the room. This limits potential experiments and slows research progress. Alternatively, trainee scientists will spend months engineering complex, automated mazes to address a specific question. Despite the time invested, these mazes are not often amenable to flexibly changing into novel maze configurations, and so the same cost in design and manufacturing must be incurred again for the next experiment.

To address these issues, we have developed a maze system that uses standardized track pieces to create flexible and scalable maze environments. The maze can be rapidly adapted to facilitate experiments that use multiple configurations, switching between shapes on the scale of minutes. Reward wells are integrated into the track pieces and support automated lick detection and reward delivery. Automated movable barriers can be placed between any track components, allowing additional environmental manipulations even during behavior. This maze system enables behavioral and neurophysiology experiments not currently viable with single behavioral tracks, all without the costs that come with custom behavioral track design. This will allow researchers to complete better experiments faster, with greater reproducibility and repeatability, and at lower cost, supporting the advance of our understanding of rodent behavior and neurophysiology.

\section{Results}

We designed a maze system using modular track pieces (Figure $1 \mathrm{~A}$ ) that enables the creation of a wide array of two-dimensional track environments (Figure 1B). Track pieces 


\section{A}

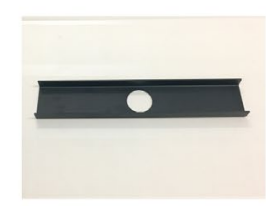

Straight

B

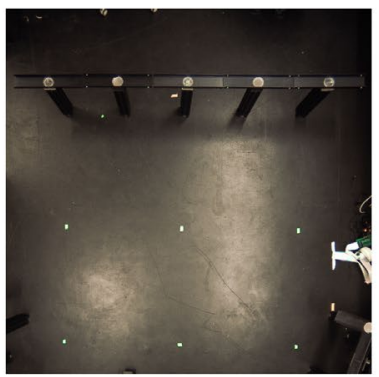

Linear

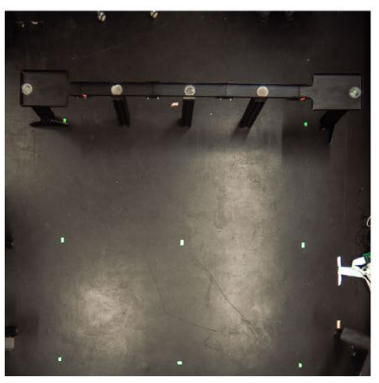

Platform Linear

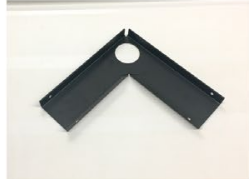

L

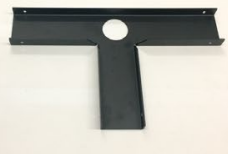

T

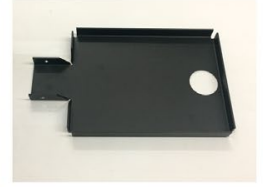

Platform

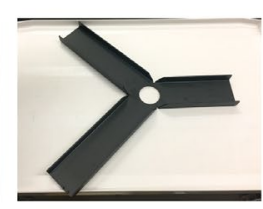

$\mathbf{Y}$

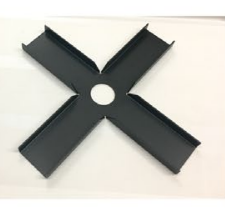

$+$

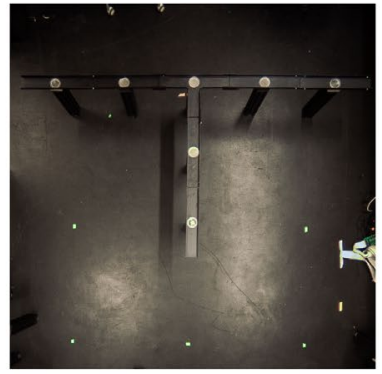

$\mathbf{T}$

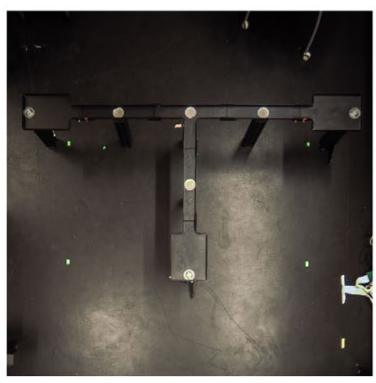

Platform T

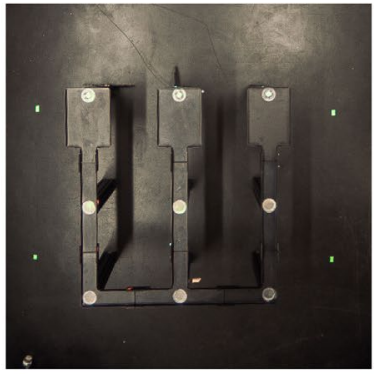

W

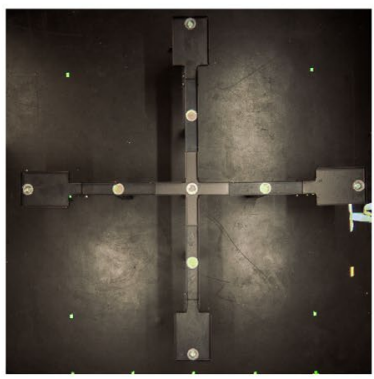

$+$

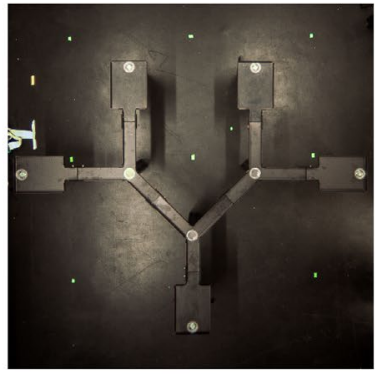

Double $Y$

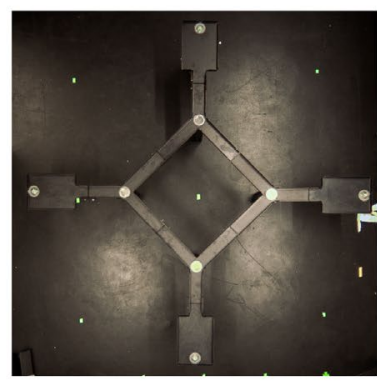

Restaurant Row

Figure 1: Adaptable Maze System. A) Mazes are constructed by combining track pieces into the desired shapes. Pictured are the six most common track piece shapes. B) Eight example mazes, including common mazes from the neurophysiology literature.

are supported individually and then connected using custom 3D-printed track joints. Track pieces are designed to meet at locations on an 18" grid (except for the platform piece, which is extended to best use the grid space), thereby allowing any combination of pieces to be used to create the maze of choice. The system is scalable, as mazes can be appended to by simply adding another piece. It is also flexible, as a maze can be adapted by replacing one or more pieces by simply disconnecting the joint connections and placing alternate track pieces. Due to the standardized design and componentry, maze environments can be easily recreated both in and across labs, greatly enhancing repeatability and reproducibility of experiments.

The maze system also features automated reward well lick detection and liquid reward distribution (Figure 2). Each track piece is connected through a reward well or plug insert and screw in connector (Figure 2A-B) to the supporting leg assembly (Figure 2C). 
Integrated into the well is an infrared beambreak circuit, two signaling LEDs, and tubing for liquid reward. These components are tucked into the leg assembly upon construction (Figure 2D), protecting componentry from curious rodents and allowing for quick connections and easy cleaning. Recording of behavior and reward control is achieved through hardware integration into the SpikeGadgets Environmental Control Unit (ECU), although any system with digital outputs could easily be accommodated. Custom software controls the ECU for reward programs for different maze layouts and reward schedules.

To achieve environmental flexibility within a recording session, the maze system includes the ability to place automated barriers between any two track pieces (Figure 3). Barriers start below the track and are raised into position through the track joint by an Arduino-driven stepper motor. Like the reward system, barrier position is controlled through digital inputs from the ECU.

\section{Discussion}

Here we have detailed a novel modular maze system for behavior and systems neuroscience research in rodents. The design integrates reward ports into the track pieces and includes automated lick detection and liquid reward distribution. Automated barriers can be included between any two track pieces, furthering flexibility of the design. Together, this design comprises a simplified system capable of enabling repeatable standard maze configurations as well as novel experimental designs.

Notably, this design allows for the construction of stereotyped and clearlydocumented maze designs within and across labs, increasing repeatability and reproducibility of results. For example,
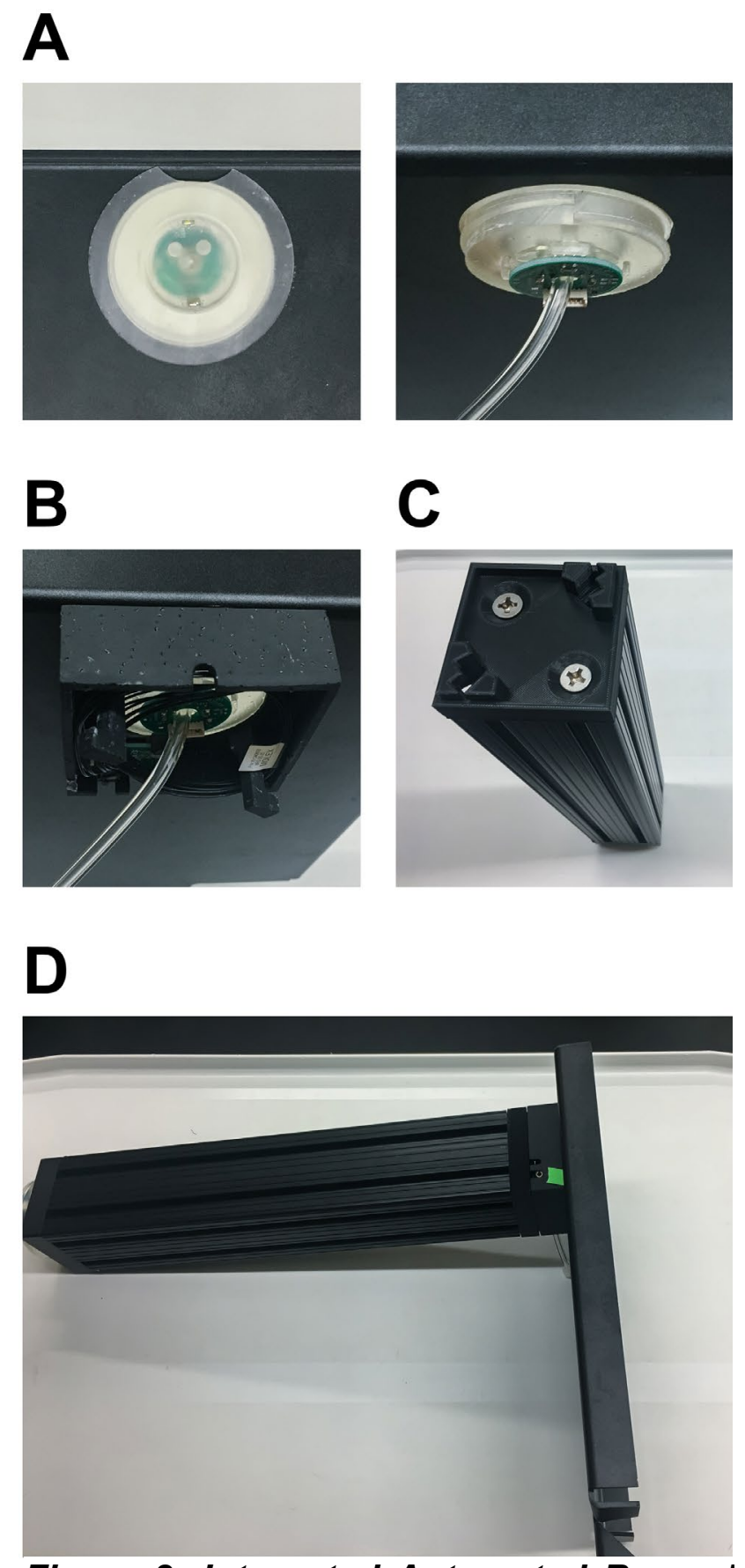

Figure 2: Integrated Automated Reward Wells. A) Reward well in a track piece from a top (left) and bottom (right) vantage point. B) Connector piece screwed onto the reward well. C) Leg Assembly with quick hooks for attachment of the track piece assembly. D) Full modular segment after the track assembly is attached to the leg assembly. Note all electronics are enclosed with exposed plugs for easy connections. 
classic spatial navigation and working memory assays on environments such as 'T', '+', and 'W' mazes can be constructed with this system to exact specifications and with shared control code. These same setups can then be exactly replicated without variability in implementation due to varied hardware (e.g. material, port type) or software.

Another benefit of a modular system is the flexibility to reuse spaces and adapt maze designs with ease. A common maze setup is a single-piece or fixed design. For another researcher to then use that same experimental space with a different maze design, the first maze must be relocated. Obviously, this limits the feasible number of actual mazes used in lab. If two or more mazes want to be used for the same experiment session, it may be difficult to make the transition in a timely manner. With our system, this can be addressed quickly and easily with the repositioning or exchanging of track pieces, facilitating both minor space manipulation experiments or complete track redesigns in minutes. This also facilitates simple scaling of mazes by adding segments between track pieces, something impossible with fixed or single-piece designs.

Perhaps the most exciting benefit of this system is the reduced engineering and setup costs of experiments. This will save months or even years for the setup of unique maze designs, and will even enable rapid prototyping of novel experiments. Because of the minimal cost of any particular configuration, many configurations can be quickly piloted to find the preferred environment design.

This maze system is completely compatible with behavioral recording as well as neurophysiology recording techniques, including in vivo electrophysiology and onephoton calcium imaging. System components are entirely below the track surface, clearing the space above the track for unimpeded visual recording or physical tethering. In particular, this design will enable continuous neural monitoring during behavioral tasks in multiple maze environments.

The design decisions of our system also come with limitations. Simple translations or rotations of the maze in the room are difficult with the current design, as the connections

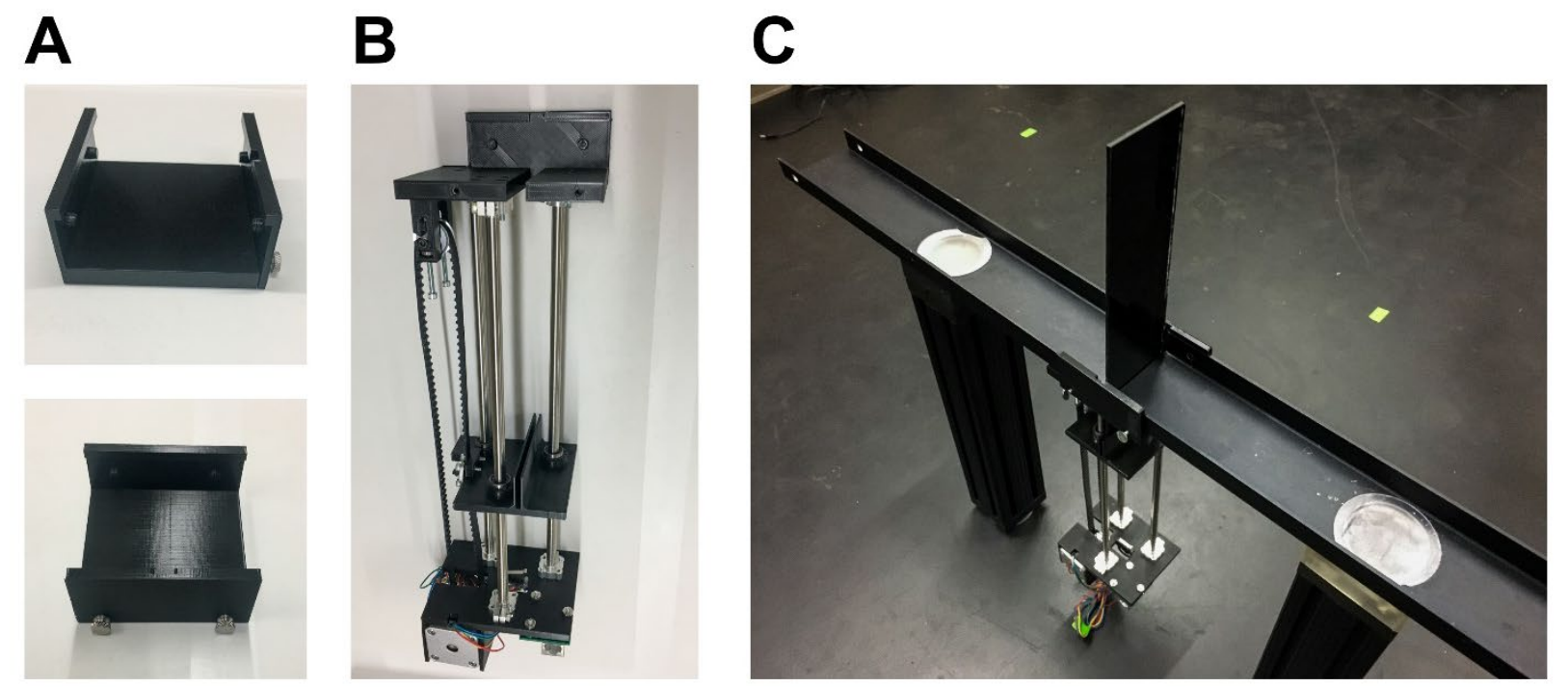

Figure 3: Automated Barriers. A) 3D-printed track joints quickly snap then screw together to lock track pieces into position. B) An automated barrier can be inserted in place of any track joint. C) An example of the automated barrier between two track pieces. 
between track segments are weak compared to the weight of the legs. One possible solution is to connect the legs with additional T-slotted beams to add strength, but this will trade off with the amount of time to adapt the system. Another concern is the extent of design flexibility. Our maze system is currently laid out on a grid system for ultimate flexibility. This does, however, limit the potential maze designs. For example, our current setup does not accommodate a classic 8 arm maze (Olton \& Samuelson, 1976). This is easily rectified however, through manufacturing the correct center piece. One can easily add additional track pieces or customize components to add functionality to the system, as the base compatibility requirements are only to interface with either our track joint and reward well designs or the basic sheet metal track pieces. Finally, this maze system is incapable of three-dimensional maze designs, so unique mazes (Grieves et al., 2020; Wilson et al., 2015) will still be required to explore three-dimensional navigation.

There are many alternative maze possibilities with different relative costs and benefits. Single-piece mazes of materials such as wood are quick and cheap to construct, but are permanent in their design and occupy considerable experimental space when not in use. Complex, unique mazes can be perfectly catered to an experiment, but often have immense engineering setup costs and lock in designs. Another maze alternative is a virtual reality (VR) setup. The utility of VR setups as compared to our design is similar to other real world mazes (Chen et al., 2018; Minderer et al., 2016). VR offers the ability to precisely control sensory variables and even decouple typically-coupled variables such as movement speed and visual flow, but that same decoupling can also be a disadvantage and creates an unnatural situation that may not be desired for the study. VR also enables head-fixed recording techniques, but requires head-fixation of animals during any experiment. Finally, an impressive modular track system was recently published by Hoshino and colleagues (Hoshino et al., 2020). Their system boasts many of the features of our system, but with a few important distinctions. Their reward system is for solid food as opposed to liquid, and their barrier system is not automated. Hoshino et al. also use a grid system but it is implemented on the floor, limiting their ability for configurations to 45 and 90 degree segments. They have developed a beambreak system that is decoupled from reward locations, allowing for more flexible behavioral control than currently available with this system. Many of these variations could be added to our system due to its fundamental simplicity, but they are not currently integrated.

Mazes remain a ubiquitous tool in rodent behavior and systems neuroscience. We have designed a modular maze system to enable flexible maze designs and rapid experiment prototyping and development. Adaptation of this standardized system will also allow for improvements in repeatability and reproducibility. Overall, the authors believe this maze system adds an important tool for researchers and will facilitate and expedite novel behavior and systems neuroscience discoveries.

\section{Materials Availability}

Resources will be very gladly shared by the authors upon request. Since development and refinement is ongoing, please contact the authors (jmolson@brandeis.edu) for inquiries regarding the maze system and access to up-to-date designs, software, and materials. 


\section{Acknowledgements}

This work was supported by a Smith Foundation Odyssey Award to S.P.J. and a Brandeis Innovation SPROUT grant. J.M.O and J.H.B were supported by NIH NINDS T32 (NS 7292-33). J.M.O. was also supported by the Swartz Foundation. We thank the Brandeis NSF I-Corps Program for guidance during product development, our I-Corps teammates Xin Yao Lin and Faye Raymond for helpful discussions during development, Francisco Mello for his assistance in fabrication of maze components, and all members of the Jadhav lab for helpful comments during the design and implementation of the maze system. 


\section{Methods}

Animal Surface. Animals move on an interlocking system of custom anodized aluminum track pieces to form the maze environment. Track pieces are all 3 " $(7.6 \mathrm{~cm})$ wide with $7 / 8$ " $(2.2 \mathrm{~cm})$ walls with variable shapes and lengths designed for an 18 " grid. Track pieces are connected using custom 3D-printed (Ender3 Pro) polylactic acid (PLA) plastic track joint connectors (Figure $3 \mathrm{~A})$ with 0.25 " $(0.6 \mathrm{~cm})$ gaps between pieces to allow for barrier insertion. Walls on interior and exterior corners are cut according to sheet metal manufacturing constraints. Each track piece has a 2.5 " $(6.4 \mathrm{~cm})$ hole for insertion of a custom reward well or plug (Figure $2 \mathrm{~A}$ ) manufactured in-house using stereolithography (SLA) 3D-printing (Form2 and Form3 printers with clear resin, FormLabs).

Support System. Each individual track piece integrates a reward well or plug and necessary electronics into a track piece assembly and is supported by its own leg assembly (Figure 2F) using a custom quick-lock system (Figure 2B).

The leg is assembled from a floor guide (part\# 2189, 8020 Inc) attached to a 18" tall 3"x3" T-slotted beam (part\# 3030, 8020 Inc) using a base plate (part\# 2140, 8020 Inc). A custom PLA base plate (Figure $2 \mathrm{C}$ ) is attached to the top of the T-slotted beam to complete the leg assembly.

The track assembly is constructed by inserting a reward well or plug through the track piece and screwed onto the custom SLA-printed connector piece (Figure 2B). The track assembly can then be installed onto the leg using the quick-lock system, which requires insertion of the connector hooks into the base plate, a 10-degree twist into position, and a press down to lock onto the base plate.

Automated Reward System. An infrared beambreak is integrated into the well to detect licks, and tubing is connected to the bottom of the well to deliver liquid reward. Port entry detection and reward delivery are automated using custom hardware and software connected into the SpikeGadgets environmental control unit. This setup allows for precise control of custom experimental setups using high level programming languages such as python or Matlab.

Automated Barriers. Automated barriers can be integrated into the maze environment between any two track pieces for within-experiment adaptation of the environment (Figure 3). Barriers are integrated into the track joint pieces and rise from between the two pieces using a stepper motor. Barriers are controlled by custom hardware and software connected into the SpikeGadgets environmental control unit, again allowing for precise control of custom experimental setups using python or Matlab. 


\section{References}

Ainge, J. A., Meer, M. A. A. van der, Langston, R. F., \& Wood, E. R. (2007). Exploring the role of context-dependent hippocampal activity in spatial alternation behavior. Hippocampus, 17(10), 988-1002. https://doi.org/10.1002/hipo.20301

Barnes, C. A. (1979). Memory deficits associated with senescence: A neurophysiological and behavioral study in the rat. Journal of Comparative and Physiological Psychology, 93(1), 74-104. https://doi.org/10.1037/h0077579

Böhm, C., \& Lee, A. K. (2020). Canonical goal-selective representations are absent from prefrontal cortex in a spatial working memory task requiring behavioral flexibility. ELife, 9, e63035. https://doi.org/10.7554/eLife.63035

Chen, G., King, J. A., Lu, Y., Cacucci, F., \& Burgess, N. (2018). Spatial cell firing during virtual navigation of open arenas by head-restrained mice. ELife, 7, e34789. https://doi.org/10.7554/eLife.34789

Frank, L. M., Brown, E. N., \& Wilson, M. (2000). Trajectory encoding in the hippocampus and entorhinal cortex. Neuron, 27(1), 169-178.

Grieves, R. M., Jedidi-Ayoub, S., Mishchanchuk, K., Liu, A., Renaudineau, S., \& Jeffery, K. J. (2020). The place-cell representation of volumetric space in rats. Nature Communications, 11(1), 789. https://doi.org/10.1038/s41467-020-14611-7

Handley, S. L., \& Mithani, S. (1984). Effects of alpha-adrenoceptor agonists and antagonists in a maze-exploration model of 'fear'-motivated behaviour. NaunynSchmiedeberg's Archives of Pharmacology, 327(1), 1-5. https://doi.org/10.1007/BF00504983

Hoshino, S., Takahashi, R., Mieno, K., Tamatsu, Y., Azechi, H., Ide, K., \& Takahashi, S. (2020). The Reconfigurable Maze Provides Flexible, Scalable, Reproducible, and $\begin{array}{llll}\text { Repeatable Tests. } & \text { IScience, } & \text { 23(1), }\end{array}$ https://doi.org/10.1016/j.isci.2019.100787

Knierim, J. J., McNaughton, B. L., \& Poe, G. R. (2000). Three-dimensional spatial selectivity of hippocampal neurons during space flight. Nature Neuroscience, 3(3), 209-210. https://doi.org/10.1038/72910

Minderer, M., Harvey, C. D., Donato, F., \& Moser, E. I. (2016). Virtual reality explored. Nature, 533(7603), 324-325. https://doi.org/10.1038/nature17899

Morris, R. G. M., Garrud, P., Rawlins, J. N., \& O’Keefe, J. (1982). Place navigation impaired in rats with hippocampal lesions. Nature, 297(5868), 681-683. https://doi.org/10.1038/297681a0

Nitz, D. A. (2006). Tracking route progression in the posterior parietal cortex. Neuron, 49(5), 747-756. https://doi.org/10.1016/j.neuron.2006.01.037

O'Keefe, J., \& Dostrovsky, J. (1971). The hippocampus as a spatial map. Preliminary evidence from unit activity in the freely-moving rat. Brain Research, 34(1), 171175. https://doi.org/10.1016/0006-8993(71)90358-1 
Olson, J. M., Tongprasearth, K., \& Nitz, D. A. (2017). Subiculum neurons map the current axis of travel. Nature Neuroscience, 20(2), 170-172. http://10.0.4.14/nn.4464

Olton, D. S., \& Samuelson, R. J. (1976). Remembrance of places passed: Spatial memory in rats. Journal of Experimental Psychology: Animal Behavior Processes, 2(2), 97116. https://doi.org/10.1037/0097-7403.2.2.97

Porter, B. S., Schmidt, R., \& Bilkey, D. K. (2018). Hippocampal place cell encoding of sloping terrain. Hippocampus, 28(11), 767-782. https://doi.org/10.1002/hipo.22966

Small, W. S. (1901). Experimental study of the mental process of the rat. II. The American Journal of Psychology, 12(2), 206-239. https://doi.org/10.2307/1412534

Steiner, A. P., \& Redish, A. D. (2014). Behavioral and neurophysiological correlates of regret in rat decision-making on a neuroeconomic task. Nature Neuroscience, 17(7), 995-1002. https://doi.org/10.1038/nn.3740

Tanila, H., Ku, S., Kloosterman, F., \& Wilson, M. A. (2018). Characteristics of CA1 place fields in a complex maze with multiple choice points. Hippocampus, 28(2), 81-96. https://doi.org/10.1002/hipo.22810

Tolman, E. C., Ritchie, B. F., \& Kalish, D. (1946). Studies in spatial learning. I. Orientation and the short-cut. Journal of Experimental Psychology: General, 36, 13-24. https://doi.org/10.1037/0096-3445.121.4.429

Wilson, J. J., Harding, E., Fortier, M., James, B., Donnett, M., Kerslake, A., O'Leary, A., Zhang, N., \& Jeffery, K. (2015). Spatial learning by mice in three dimensions. Behavioural Brain Research, 289, 125-132. https://doi.org/10.1016/j.bbr.2015.04.035 